\title{
Using Lean Quality Improvement Tools to Increase Delivery of Evidence-Based Tobacco Use Treatment in Hospitalized Neurosurgical Patients
}

\author{
Laurel Sisler, MSW, CTTS; Oluwaseun Omofoye, MD, MS; Karina Paci, BS; Eldad Hadar, MD; Adam O. Goldstein, \\ MD, MPH; Carol Ripley-Moffitt, MDiv, CTTS
}

Background: Health care providers routinely undertreat tobacco dependence, indicating a need for innovative ways to increase delivery of evidence-based care. Lean, a set of quality improvement (QI) tools used increasingly in health care, can help streamline processes, create buy-in for use of evidence-based practices, and lead to the identification of solutions on the basis of a problem's root causes. To date, no published research has examined the use of Lean tools in tobacco dependence. A 12-month QI project using Lean tools was conducted to increase delivery of evidence-based tobacco use treatment (TUT) to hospitalized neurosurgical patients.

Methods: The study team developed a nicotine replacement therapy (NRT) and counseling protocol for neurosurgery inpatients who indicated current tobacco use and used Lean tools to increase protocol adherence. Rates of NRT prescription, referrals to counseling, and follow-up phone calls were compared pre- and postintervention. Secondary measures included patient satisfaction with intervention, quit rates, and reduction rates at 4 weeks postdischarge.

Results: Referrals to counseling doubled from $31.7 \%$ at baseline to $62.0 \%$ after implementation of the intervention, and rates of nicotine replacement therapy (NRT) prescriptions during hospitalization and at discharge increased from $15.3 \%$ to $28.5 \%$ and $9.0 \%$ to $19.3 \%$, respectively. Follow-up phone call rates also dramatically increased. The majority of satisfaction survey respondents indicated that counseling had a positive or neutral impact on stress level and overall satisfaction.

Conclusion: Lean tools can dramatically increase use of evidence-based TUT in hospitalized patients. This project is easily replicable by professionals seeking to improve delivery of tobacco treatment. These findings may be particularly helpful to inpatient surgical departments that have traditionally been reticent to prescribe NRT.

T obacco use is the leading preventable cause of morbidity and mortality across the globe, causing approximately six million deaths each year. ${ }^{1,2}$ Evidence-based tobacco use treatment (TUT) that includes behavioral counseling and pharmacotherapy is highly effective and affordable in both inpatient and outpatient settings. ${ }^{3-6}$ These interventions may greatly reduce tobacco-related illness and death. ${ }^{5}$ Yet health care providers routinely undertreat tobacco dependence. ${ }^{7}$ The need for innovative ways to increase delivery of evidencebased care is urgent.

Clinical practice guidelines assert the benefits of combining counseling with pharmacotherapy to optimize tobacco quit rates. ${ }^{6,8,9}$ A 2012 Cochrane review of TUT for hospitalized patients concludes that interventions beginning in the hospital and including at least one month of follow-up are most effective. ${ }^{10}$ For the purpose of this article, evidencebased care includes clinical practice guidelines and the Cochrane review recommendations. Implementation of an "opt-out" automated and universal TUT inpatient program consistent with The Joint Commission's Tobacco Treatment (TOB) performance measures for hospitals ${ }^{11}$ has proven to be feasible. ${ }^{12,13}$ A study in Australia showed that the provision of evidence-based TUT to inpatients increased after implementation of a multifaceted quality initiative but that only $21.4 \%$ of patients with tobacco dependence received advice to quit, which represents the lowest level of quality care, highlighting the need for additional strategies to optimize care delivery. ${ }^{14}$ Many hospitals in Canada ${ }^{15}$ and England, ${ }^{16}$ for example, routinely offer universal TUT counseling and nicotine replacement therapy (NRT); however, this practice is rare in the United States and other countries.

While strong evidence supports the efficacy of addressing tobacco use in hospitalized patients, the many competing demands of acute care in inpatient settings often place it at a lower priority for busy providers. ${ }^{17}$ Quality improvement (QI) techniques adopted from other industries are now widely used in health care to address gaps in quality care delivery and may be helpful in increasing the provision of evidencebased TUT to hospitalized patients.

Lean QI strategies, originally developed by the Toyota Motor Company, emphasize using the scientific method (A3 problem solving) to involve frontline providers in solving their problems related to work flow and quality care delivery, with a focus on increasing value delivered to the customer. ${ }^{18}$ Lean philosophy focuses on customers, who may be patients, providers, or other stakeholders, depending on the process under examination. ${ }^{19}$ The $\mathrm{A} 3$ consists of nine boxes or steps to plan, execute, and evaluate experiments, ${ }^{20,21}$ which were used to 


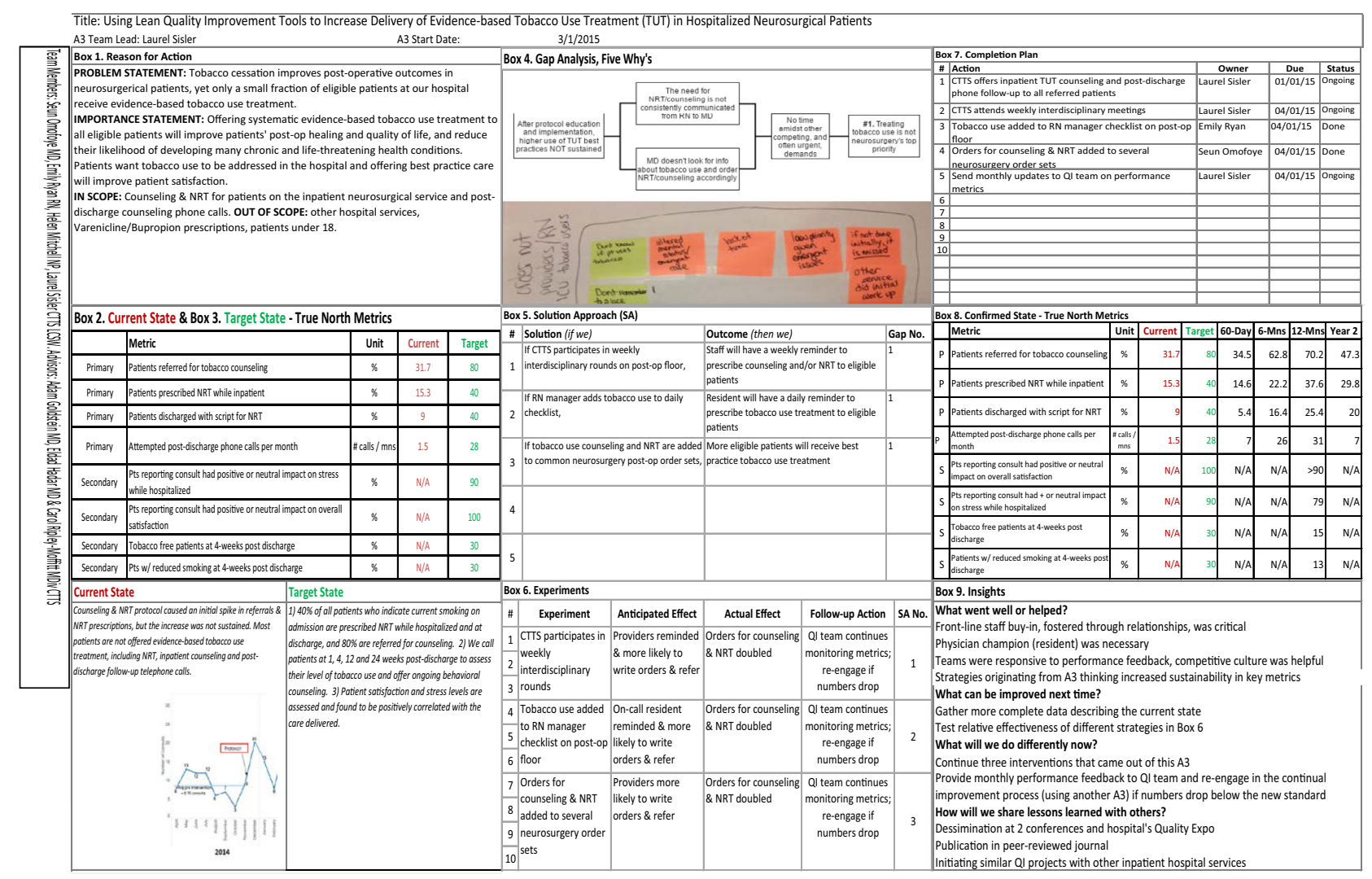

Figure 1: The $A 3$, as shown, depicts in detail the quality improvement process used by the study team. The A3 consists of nine boxes or steps to plan, execute and evaluate experiments, which were used to structure the project. NRT, nicotine replacement therapy; CTTS, Certified Tobacco Treatment Specialist; QI, quality improvement; MNs, months.

structure this project (Figure 1). Although some studies support the use of Lean tools in health care, including application in pediatrics, ${ }^{22}$ neurosurgery, ${ }^{23}$ and radiology, ${ }^{24}$ more research is warranted to fully evaluate Lean methodology within health care settings. ${ }^{25}$

Surgical inpatient services have led the medical field in incorporating QI tools into regular work flow (for example, surgical checklists, time-outs), ${ }^{26}$ but they have been hesitant to adopt TUT protocols, primarily because of misconceptions about the safety of NRT use in surgical patients. ${ }^{27}$ To our knowledge, no literature exists regarding use of Lean QI tools within the field of TUT. In this article, we report findings from a 12-month QI project using Lean tools to optimize delivery of TUT on an inpatient neurosurgery service within one institution.

\section{METHODS}

\section{Setting}

This one-year study (January 1, 2015-December 31, 2015) was conducted at a large academic medical center in the southeastern United States in which tobacco treatment specialists (TTSs) provide bedside consults to hospitalized patients and postdischarge telephone counseling as part of a comprehensive tobacco treatment service. Study \#15-3324
(Principal Investigator, Oluwaseun Omofoye) was approved by the Institutional Review Board of the University of North Carolina at Chapel Hill.

We now describe the study in terms of its phases, with a focus on (1) the introduction of a protocol with guidelines for referrals to counseling, prescription of NRT and post-discharge telephone counseling creation and postdischarge telephone counseling and (2) the subsequent application of Lean QI tools to advance implementation of the protocol.

\section{Introduction of the Protocol}

In an April 2014 presentation at Neurosurgery Grand Rounds, two TTSs [L.S., A.O.G.], emphasized the safety of NRT and the benefits of cessation to neurosurgical patients. They also provided performance feedback regarding the service's use of evidence-based cessation practices - data for the JanuaryMarch 2014 period indicated that only one third of neurosurgical patients who smoke were referred for counseling and that fewer patients received NRT. A neurosurgery resident [O.O.] volunteered to work with the TTSs on developing a protocol aimed at improving delivery of evidencebased care, and they recruited other frontline providers (a nurse practitioner and nurse manager) to form a 

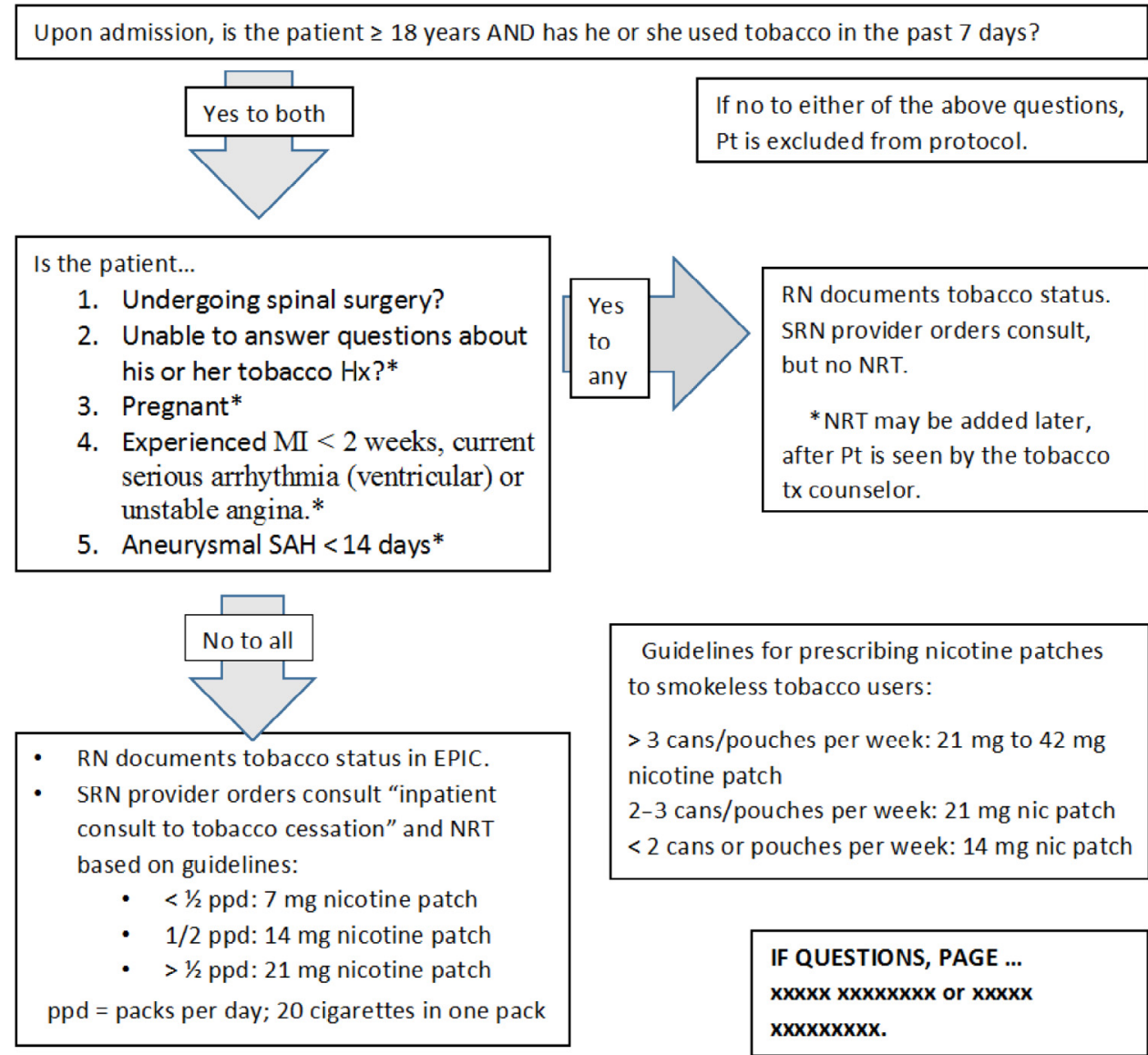
Guidelines for prescribing nicotine patches to smokeless tobacco users:
$>3$ cans/pouches per week: $21 \mathrm{mg}$ to $42 \mathrm{mg}$ nicotine patch
2-3 cans/pouches per week: $21 \mathrm{mg}$ nic patch $<2$ cans or pouches per week: $14 \mathrm{mg}$ nic patch

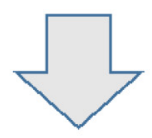

Tobacco Treatment Counselor sees patient and, a) Reassesses NRT dosing and recommends changes to medical team if necessary (shortacting NRT added as needed)

b) Engages Pt in tobacco treatment counseling

IF QUESTIONS, PAGE ...

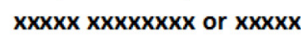
$x x x x x x x x x$.
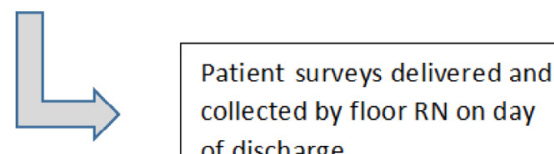

Figure 2: This protocol guided the prescription of nicotine replacement therapy and provision of tobacco use treatment counseling and follow-up in neurosurgical patients, January 2015 onward. Pt, patient; Hx, history; MI, myocardial infarction; SAH, subarachnoid hemorrhage; SRN, neurosurgery.

multidisciplinary team. The study team's Reason for Action is shown in Figure 1 (Box 1). Between April and December 2014, the team created, marketed, and disseminated a new protocol with guidelines for referrals to counseling, prescription of NRT, and postdischarge telephone counseling (Figure 2). Providers were educated about the protocol in staff meetings, huddles, and Grand Rounds. The use of the protocol appeared to result in a short-term increase in referrals for counseling and rates of NRT prescription. However, the change was not sustained beyond two months. Several team members had participated in the Lean training offered by the health care system, and in March 2015 the team 
decided to test Lean tools in the context of neurosurgical inpatient TUT.

\section{Application of the Lean QI Tools}

Following the $\mathrm{A} 3$ problem-solving tool, the team first mapped out its Current and Future Desired (Target) States (Boxes 2 and 3, Figure 1). Then, in examining the gap between the Current and Future Desired State, the team used the "Five Whys"; that is, asked why the gap exists five consecutive times to determine root causes for the gap between evidencebased practice and current state (Box 4, Figure 1). ${ }^{28}$ The team identified one likely root cause-treating tobacco dependence is not neurosurgeons' primary job. Frequently, neurosurgeons have urgent clinical issues that absorb their time, so preventive measures such as cessation services for tobacco use, albeit important, are not priorities for them.

The frontline neurosurgery providers demonstrated commitment to helping their patients with tobacco dependence by fully supporting this QI project, but they requested assistance in remembering to order evidence-based treatment. With this root cause and input from stakeholders in mind, the study team then tested three solution strategies (Boxes 5 , 6 , and 7, Figure 1) aimed at delivering better care to hospitalized patients who smoke: (1) TTS attendance at weekly interdisciplinary unit meetings, (2) tobacco use added to checklist reviewed by resident and nurse manager each morning, and (3) NRT and order for TTS consult added to multiple neurosurgery order sets.

\section{Participants}

During the study year (January 1, 2015-December 31, 2015), 337 patients 18 years of age or older who indicated current tobacco use (that is, in the 30 days prior to admission) were admitted to the inpatient neurosurgical service. The average age of the study patients was 49 years, with the majority (66\%) of patients falling between 40 and 70 years of age. The historical control group consisted of 189 neurosurgical patients who indicated tobacco use on admission between July 1, 2014, and December 31, 2014. The average age of the study patients in the control group was 52 years, with the majority $(68 \%)$ of the patients falling between 40 and 70 years of age. Some 342 patients meeting the same criteria were admitted in 2016, and their data were included to report on sustainability. The participant flow from admission to follow-up is shown in Figure 3.

\section{Primary Measures}

The team monitored rates of NRT prescription (both during hospitalization and at discharge), counseling referrals, and average follow-up phone calls attempted per month from July 1, 2014, to December 31, 2016. At four weeks

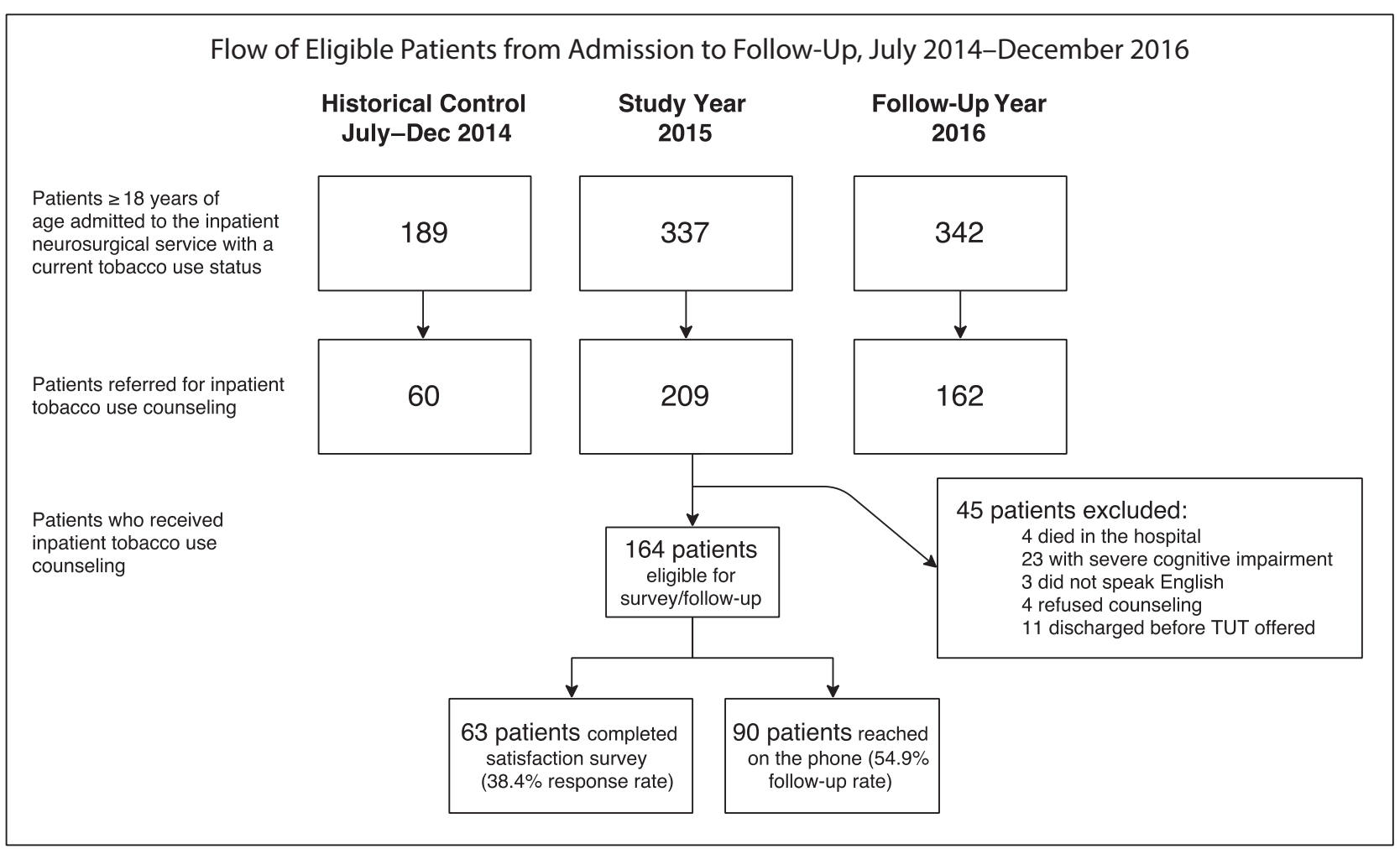

Figure 3: During the study year (January 1, 2015-December 31, 2015), 337 patients $\geq 18$ years of age, who indicated current tobacco use (that is, in the 30 days prior to admission) were admitted to the inpatient neurosurgical service. The historical control group consisted of 189 neurosurgical patients who indicated tobacco use on admission between July 1, 2014, and December 31, 2014. TUT, tobacco use treatment. 
postdischarge, study personnel made three attempts to reach every patient who received counseling while hospitalized $(N=164)$ for postdischarge follow-up telephone counseling. The measures ("Metrics") are shown in Box 2, Figure 1. Patients with certain diagnoses were excluded from receiving NRT, as shown in Figure 2, but were still included in the denominator because diagnosis information was not in our data set.

\section{Measures}

Primary Measures. We chose to primarily measure processes (referrals, prescriptions, attempted calls) rather than outcomes (receipt of counseling, administration of NRT, quit rates) because research had shown that implementing these processes results in improved patient outcomes. ${ }^{6,10}$

Secondary Measures. The quit and reduction rates were calculated as secondary outcomes data (Box 2, Figure 1) collected during follow-up calls. Also, we developed a penand-paper patient satisfaction survey, which was given on the day of discharge (Appendix 1, available in online article). The survey assessed the acceptability of universal TUT counseling from the patient perspective, in line with Lean's emphasis on delivering value to the patient. It also addressed the concerns of several neurosurgeons that TUT counseling might increase stress levels and reduce satisfaction among their patients. Floor nurses delivered the survey and collected it prior to discharge. Study personnel collected surveys weekly.

\section{Data Analysis}

Health care system analysts developed a report on rates of NRT prescription and referral to counseling with input from the study team and data from the electronic health record. This report was saved on a secure shared drive and re- viewed by study personnel each month. Study personnel used Microsoft Excel (Microsoft Corp., Redmond Washington) to compute descriptive statistics, comparing aggregated data from the historical control to the intervention period. Outcome data for the primary measures from the year after the intervention were also included to demonstrate sustainability. Team members manually entered survey responses into and analyzed aggregated data in Qualtrics (Provo, Utah), a secure Internet-based survey tool.

\section{RESULTS}

\section{Primary Measures}

As shown in Box 8 (Figure 1), following implementation of Lean tools and the resulting strategies, utilization of all evidence-based TUT practices doubled. Sixty-two percent (209) of the 337 neurosurgical patients who smoke were referred for counseling postintervention, compared to 60 of 189 (31.7\%) preintervention. Similarly, rates of inpatient NRT prescription increased from $15.3 \%$ (29 of $189 \mathrm{pa}-$ tients) to $28.5 \%$ (96 of 337), and the rates of discharge NRT prescription increased from $9.0 \%$ (17 of 189) to $19.3 \%$ (65 of 337) (Figure 4). During the study year, an average of 31 follow-up phone calls were attempted per month, compared to 1.5 per month preintervention. In the subsequent year, high NRT prescription rates were sustained $29.8 \%$ for inpatient and 20\% for discharge). Referrals for counseling and follow-up phone calls rates reduced to $47.3 \%$ (still up nearly $50 \%$ from preintervention) and 7 per month, respectively.

\section{Secondary Measures}

Patient Satisfaction Survey. Over the study period, 209 of the 337 patients with current tobacco use status were referred for counseling. Of these 209 patients, 45 did not receive counseling because they died prior to discharge $(n=4)$, were

\section{Percentage of Neurosurgery Tobacco Users Receiving Evidence-Based Treatment, Pre- vs. Postintervention}

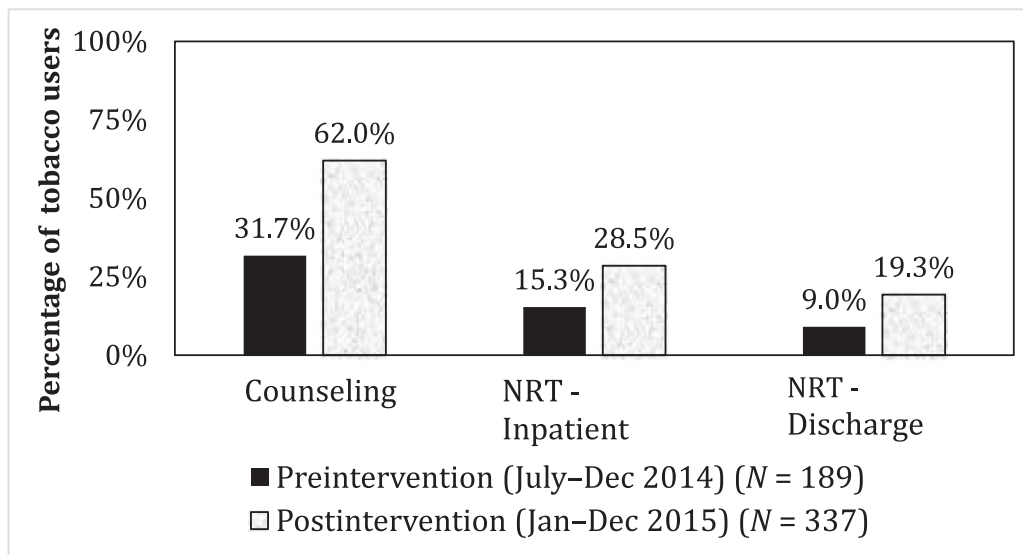

Figure 4: The graph shows the change in counseling referrals and rates of nicotine replacement therapy (NRT) prescription, pre- vs. postintervention for three of four primary measures. For example, 209 (62.0\%) of the 337 neurosurgical patients who smoke were referred for counseling postintervention, compared to 60 (31.7\% of 189 patients) preintervention. 
too cognitively impaired $(n=23)$, did not speak Englishthe survey was not translated by the hospital's translation services in time for its use in the study period. $(n=3)$, refused counseling $(n=4)$, or were discharged prior to completion of counseling consult $(n=11)$. Of the remaining $164 \mathrm{pa}-$ tients who were eligible to receive the survey, 64 completed it. One respondent did not remember meeting the TTSs, resulting in 63 complete surveys (38.4\% response rate). More than $90 \%$ of the respondents indicated that the counseling had a positive or neutral impact on their overall satisfaction with their hospital stay, $89 \%$ were satisfied with the counseling provided, $79 \%$ indicated that the counseling decreased or had no impact on their stress level during their hospital stay," and $73 \%$ reported increased confidence in their ability to quit after talking with the counselor.

Quit and Reduction Rates. At four weeks postdischarge, 90 of the 164 counseled inpatients were reached on the phone (54.9\% follow-up rate), 25 of whom reported being tobacco free since discharge. An additional 21 patients had reduced their cigarette consumption by greater than half, compared to pre-hospitalization. Using intent-to-treat analysis, by which all eligible patients are included in the analysis whether reached on the phone or not, ${ }^{29}$ the quit rate was $15.2 \%$.

\section{DISCUSSION}

Inpatient tobacco cessation interventions that include at least one month of postdischarge follow-up are effective in increasing quit rates, ${ }^{10}$ yet few hospitals fully implement this type of program. A neurosurgical service within a large academic medical center in the southeastern United States identified the need to improve delivery of evidence-based TUT. We describe the successful implementation of a QI project on this service in this article, which is noteworthy, we believe, for several reasons. First, to the best of our knowledge, it is the only published description of a comprehensive successful TUT program for hospitalized neurosurgical patients. The extensive rewards to neurosurgical patients of becoming tobacco free include primary and secondary stroke prevention, reduced incidence of infections, and improved wound healing. ${ }^{30,31}$ Lean tools proved to be an effective way to increase delivery of evidence-based care to this at-risk patient group.

Second, like neurosurgeons, many other surgeons are reluctant to prescribe NRT because of concerns about the impact of NRT on vasoconstriction and wound healingconcerns that arise largely from experimental studies. ${ }^{32,33}$ Despite literature that supports the safety of NRT and demonstrates no increased risk of vasospasm or impaired wound healing, ${ }^{27,30,34-38}$ knowledge and acceptance of this literature remains low among surgeons. ${ }^{39}$ The current study demonstrates an effective means to provide surgeons and other staff with updated education about NRT, create a safe NRT protocol for surgical patients, and change culture in support of evidence-based TUT. Other surgical units may use this model for treating tobacco dependence while maintaining patient safety as a top priority.

Third, this project highlights a successful intervention delivered to hospitalized patients. For example, both inpatient and discharge NRT prescription rates doubled-to $28.5 \%$ and $19.3 \%$, respectively. These percentages would likely have been even higher, except that two categories of patients were exempt from receiving NRT - those with a subarachnoid hemorrhage (SAH) and those requiring instrumented spinal fusion. Retrospective analysis showing that NRT does not increase risk of vasospasm in patients with $\mathrm{SAH}^{34}$ did not change neurosurgeons' perspective that NRT is too risky in this vulnerable population. Preclinical data suggesting that high levels of nicotine are associated with delayed spinal fusion rates in rabbits ${ }^{40}$ and rats ${ }^{41}$ led to the decision to exclude this patient population. These two categories combined make up $54 \%$ of all neurosurgical patients at the study hospital, regardless of their smoking status, admitted in 2015. Within our study sample and control group, the percentage of patients in these excluded categories is unknown because diagnosis information was not in our study data set, but likely a substantial portion of those who did not receive NRT were ineligible for NRT.

Fourth, these data may have financial implications. Health care systems may lose money when they miss opportunities to help patients stop smoking. Evidence suggests that patients admitted for cardiovascular problems, including stroke, who continue smoking postdischarge are at increased risk of readmission. ${ }^{42-46}$ Novel strategies to increase delivery of evidence-based care are necessary, and our project suggests that Lean tools may be a promising strategy to reach this goal.

Finally, the patient satisfaction survey data were encouraging, as the TTS consults were well received, with nearly $90 \%$ of respondents reporting satisfaction with the treatment provided. While a $38.4 \%$ response rate for patient satisfaction surveys was not ideal, we identified two primary barriers to completion: the plethora of tasks requiring nurse attention prior to discharge and the inability of some patients to complete the survey. The latter reflects a real barrier to surveying patients when recent nervous system pathology results in limited cognitive capacity.

To sustain progress, the project team provides monthly performance reports to neurosurgery stakeholders on primary metrics. Staff members receive routine reminders about the new work flow at quarterly nurse staff meetings, during biannual physician Grand Rounds, and during neurosurgery intern orientation each July. This strategy has been sufficient to maintain the new status quo for NRT prescription rates. Referrals for counseling reduced slightly in 2016, likely related to fewer referrals for patients with compromised neurological status. Follow-up phone call rates consequently decreased as well. The satisfaction survey, which required considerable staff time, was not continued beyond the study year. If different implementation strategies are needed in the future, the team will start another improvement cycle using Lean 
tools. As shown in Box 9 in Figure 1, the team identified "Insights" from the project, which included testing the relative effectiveness of different strategies and initiating similar QI projects with other inpatient hospital services. In 2016, heart and vascular services initiated a similar project with positive results.

\section{Limitations}

This study was conducted on one surgical service in one hospital, so the results may not be generalizable. However, we had a relatively large sample size $(N=337)$, and the results were robust over the course of two years. Also, Lean methodology helps to account for differences by service and hospital by enabling frontline workers to devise solutions to their daily problems. In addition, we did not attempt to discern the relative effectiveness of the three chosen strategies aimed at improving delivery of evidence-based TUT. On the basis of feedback from ground-level providers, we hypothesize that the strengthened relationship between neurosurgery and the TTS consult service as a result of regular contact, coupled with daily reminders around evidencebased practices, were key factors driving the project's success. Adding evidence-based TUT practices to order sets is a "just do it" intervention because it requires no time investment after the initial change. Further research should investigate the relative effectiveness of interventions that are more time intensive, such as TTS attendance at weekly meetings versus daily checklist review between residents and nurse managers.

There were also some limitations with our data collection methods and chosen metrics. No data for secondary measures were collected during the historical control period, so that the "current state" for these measures was unknown. Also, the study's custom outcome report did not include diagnosis data, complicating determination of the denominator for NRT prescription rates, as it was impossible to exclude those ineligible for NRT per the protocol in Figure 2. In addition, the quit rate at four weeks postdischarge-15\%-was lower than that of some randomized controlled trials (RCTs), ${ }^{47,48}$ but assessing quit rates was not our primary aim. In any case, real-world unassisted quit rates within the neurosurgical population are unknown, so that finding an appropriate comparison group would have been challenging. Shi and Warner explored the number of successful quits in the United States that were attributable to various surgical procedures, but neurosurgical procedures were not included. ${ }^{49}$ Moreover, our team lacked human resources to make more than three attempted follow-up calls, and some RCTs make six attempted calls and/or use interactive voice response to reach more participants. ${ }^{12,48}$ Finally, some RCTs exclude patients with cognitive impairment and/or those admitted to the ICU, which compromises the majority of our participant sample. We attempted to improve delivery of evidence-based practices that other studies ${ }^{47,48}$ have shown improve quit rates. Further research should investigate quit rates in neurosurgical patients comparing usual care to evidence-based practice.

\section{CONCLUSION}

We have demonstrated that Lean tools can assist inpatient hospital units to increase delivery of evidence-based TUT for the reduction of tobacco-related morbidity and mortality. More research is needed to determine if this model is effective on other inpatient units and in other settings. In addition, the satisfaction data may alleviate concerns about the acceptability of universal tobacco treatment counseling among hospitalized patients who smoke.

\begin{abstract}
Acknowledgments. The authors acknowledge Jennifer Greyber for editorial assistance in preparing the manuscript for submission. They also acknowledge the North Carolina Translational \& Clinical Sciences Institute (NC TraCS) at the University of North Carolina at Chapel Hill for help with data extraction.
\end{abstract}

Conflicts of Interest. All authors report no conflicts of interest.

Laurel Sisler, MSW, CTTS, formerly Program Manager, Inpatient Tobacco Use Treatment program, University of North Carolina (UNC) Hospital, Chapel Hill, is Director, UNC Nicotine Dependence Program, Department of Family Medicine, Chapel Hill. Oluwaseun Omofoye, MD, MS, formerly Resident Physician, UNC Department of Neurosurgery, is Clinical Fellow, Boston Medical Center, Department of Neurosurgery. Karina Paci, MD Candidate, is Medical Student, UNC School of Medicine, Chapel Hill. Eldad J. Hadar, MD, is Professor, UNC Department of Neurosurgery, Chapel Hill. Adam O. Goldstein, MD, MPH, is Professor, UNC Department of Family Medicine, and Director, Tobacco Intervention Programs, UNC School of Medicine. Carol Ripley-Moffitt, MDiv, CTTS (ret.), was Director, UNC Nicotine Dependence Program, Department of Family Medicine. Please address correspondence to Laurel Sisler, laurel_sisler@med.unc.edu.

\section{ONLINE-ONLY CONTENT}

See the online version of this article for Appendix 1. Patient Satisfaction Survey Administered to Hospitalized Neurosurgical Patients with a Current Smoking Status on the Day of Discharge, January-December 2015.

\section{REFERENCES}

1. US Department of Health and Human Services, Office of the Surgeon General. The Health Consequences of Smoking-50 Years of Progress: A Report of the Surgeon General. Atlanta: Centers for Disease Control and Prevention, National Center for Chronic Disease Prevention and Health Promotion, Office on Smoking and Health. 2014. Accessed Sep 27, 2017. https://www.surgeongeneral.gov/library/reports/50-years -of-progress/full-report.pdf.

2. World Health Organization. WHO Report on the Global Tobacco Epidemic, 2011: Warning About the Dangers of Tobacco. 2011. Accessed Sep 27, 2017. http://www.who.int/ tobacco/global_report/2011/en/.

3. West R, McNeill A, Raw M. Smoking cessation guidelines for health professionals: an update. Thorax. 2000;55:987999.

4. Piper ME, et al. Identifying effective intervention components for smoking cessation: a factorial screening experiment. Addiction. 2016;111:129-141.

5. Patnode CD, et al. Behavioral counseling and pharmacotherapy interventions for tobacco cessation in 
adults, including pregnant women: a review of reviews for the US Preventive Services Task Force. Ann Intern Med. 2015 Oct 20;163:608-621.

6. Fiore M, et al. Treating Tobacco Use and Dependence: 2008 Update. Rockville, MD: US Department of Health and Human Services, Public Health Service, May 2008. Accessed Sep 20, 2017. https://www.ncbi.nlm.nih.gov/books/NBK63952/.

7. Bernstein $\mathrm{SL}$, et al. Undertreatment of tobacco use relative to other chronic conditions. Am J Public Health. 2013; 103:e59-e65.

8. Bittner JC, et al. Smoking status and smoking cessation intervention among U.S. adults hospitalized for asthma exacerbation. Allergy Asthma Proc. 2016;37:318-323.

9. Shin $\mathrm{GH}$, et al. Compliance and effectiveness of smoking cessation program started on hospitalized patients. Korean J Fam Med. 2016;37:149-155.

10. Rigotti NA, et al. Interventions for smoking cessation in hospitalised patients. Cochrane Database Syst Rev. 2012 May 16:CD001837. Accessed Sep 27, 2017. http:// onlinelibrary.wiley.com/doi/10.1002/14651858.CD001837 .pub3/pdf.

11. The Joint Commission. Specifications Manual for National Hospital Inpatient Quality Measures, v. 5.2a. Oct 20, 2016. Accessed Sep 27, 2017. https://www.jointcommission .org/assets/1/6/NEW_HIQR_SpecsMan_5_1_July2016 _PDF.zip.

12. Nahhas GJ, et al. Who opted out of an opt-out smokingcessation programme for hospitalised patients? J Smok Cessat. Epub 2016 August 31.

13. Nahhas GJ, et al. Feasibility of implementing a hospitalbased "opt-out" tobacco-cessation service. Nicotine Tob Res. Epub 2016 Dec 7.

14. Slattery $C$, et al. Increasing smoking cessation care across a network of hospitals: an implementation study. Implement Sci. 2016 Mar 1;11:28.

15. Reid RD, et al. Smoking cessation for hospitalized smokers: an evaluation of the "Ottawa Model. Nicotine Tob Res. 2010;12:11-18.

16. Bickerstaffe G. Smoking cessation for hospital inpatients. BMJ Qual Improv Rep. 2014 Jul 18;3:u204964.w2110.

17. Liu SK, et al. Improving the quality of care for the hospitalized tobacco user-one institution's transformational journey. J Cancer Educ. 2010;25:297-301.

18. Thompson DN, Wolf GA, Spear SJ. Driving improvement in patient care: lessons from Toyota. J Nurs Adm. 2003;33:585595.

19. Vest JR, Gamm LD. A critical review of the research literature on Six Sigma, Lean and StuderGroup's Hardwiring Excellence in the United States: the need to demonstrate and communicate the effectiveness of transformation strategies in healthcare. Implement Sci. 2009 Jul 1;4:35.

20. Sobek DK II, Smalley A. Understanding A3 Thinking: A Critical Component of Toyota's PDCA Management System. Boca Raton, FL: Productivity Press, 2008.

21. Mazur LM, Chen SJ, Prescott B. Pragmatic evaluation of Toyota Production System (TPS) analysis procedure for problem solving with entry-level nurses. J Ind Eng Manag. 2008;1:240-268.

22. Gleich SJ, et al. Improvement in patient transfer process from the operating room to the PICU using a Lean and Six Sigma-based quality improvement project. Hosp Pediatr. 2016;6:483-489.

23. Raskin JS, et al. Application of Lean principles to neurosurgical procedures: the case of lumbar spinal fusion surgery. Neurosurgery. 2015;62 (CN Suppl 1):212.
24. Amaratunga T, Dobranowski J. Systematic review of the application of Lean and Six Sigma quality improvement methodologies in radiology. J Am Coll Radiol. 2016;13:10881095.e7.

25. Glasgow JM, Scott-Caziewell JR, Kaboli PJ. Guiding inpatient quality improvement: a systematic review of Lean and Six Sigma. Jt Comm J Qual Patient Saf. 2010;36:533-540.

26. Cima RR, et al. Use of Lean and Six Sigma methodology to improve operating room efficiency in a high-volume tertiarycare academic medical center. J Am Coll Surg. 2011;213:8392.

27. Nolan MB, Warner DO. Safety and efficacy of nicotine replacement therapy in the perioperative period: a narrative review. Mayo Clin Proc. 2015;90:1553-1561.

28. iSixSigma®. 5 Whys. Accessed Sep 27, 2017. https:// www.isixsigma.com/tools-templates/5-whys/.

29. Gupta SK. Intention-to-treat concept: a review. Perspect Clin Res. 2011;2:109-112.

30. Møller AM, et al. Effect of preoperative smoking intervention on postoperative complications: a randomised clinical trial. Lancet. 2002 Jan 12;359:114-117.

31. Ockene IS, Miller NH, American Heart Association Task Force on Risk Reduction. Cigarette smoking, cardiovascular disease, and stroke: a statement for healthcare professionals from the American Heart Association. Circulation. 1997 Nov 4;96:3243-3247.

32. Black CE, et al. Effect of nicotine on vasoconstrictor and vasodilator responses in human skin vasculature. Am J Physiol Regul Integr Comp Physiol. 2001;281:R1097-1104.

33. Forrest $C R, X u N$, Pang $C Y$. Evidence for nicotine-induced skin flap ischemic necrosis in the pig. Can J Physiol Pharmacol. 1994;72:30-38.

34. Carandang RA, et al. Nicotine replacement therapy after subarachnoid hemorrhage is not associated with increased vasospasm. Stroke. 2011;42:3080-3086.

35. Seder DB, et al. Transdermal nicotine replacement therapy in cigarette smokers with acute subarachnoid hemorrhage. Neurocrit Care. 2011;14:77-83.

36. Sørensen LT. Wound healing and infection in surgery: the pathophysiological impact of smoking, smoking cessation, and nicotine replacement therapy: a systematic review. Ann Surg. 2012;255:1069-1079.

37. Sorensen LT, Karlsmark T, Gottrup F. Abstinence from smoking reduces incisional wound infection: a randomized controlled trial. Ann Surg. 2003;238:1-5.

38. Sisler $\mathrm{L}$, et al. Case report: nicotine replacement therapy (NRT) in hospitalized surgical patient. J Smok Cessat. 2015;10:2-4.

39. Warner DO, et al. Anesthesiologists, general surgeons, and tobacco interventions in the perioperative period. Anesth Analg. 2004;99:1766-1773.

40. Silcox DH 3rd, et al. The effect of nicotine on spinal fusion. Spine. (Phila PA 1976).1995 Jul 15;20:1549-1553.

41. Skott $M$, et al. Tobacco extract but not nicotine impairs the mechanical strength of fracture healing in rats. J Orthop Res. 2006;24:1472-1479.

42. Lightwood JM, Glantz SA. Short-term economic and health benefits of smoking cessation myocardial infarction and stroke. Circulation. 1997 Aug 19;96:1089-1096.

43. Mohiuddin SM, et al. Intensive smoking cessation intervention reduces mortality in high-risk smokers with cardiovascular disease. Chest. 2007;131:446-452.

44. Lightwood J, Fleischmann KE, Glantz SA. Smoking cessation in heart failure: it is never too late. J Am Coll Cardiol. 2001;37:1683-1684. 
45. Cartmell KB, et al. Reducing hospital readmission rates by implementing an inpatient tobacco dependence treatment service. Poster session presented at the National Conference on Tobacco or Health, Austin, TX, Mar 2017.

46. Cartmell KB, et al. Reducing healthcare costs by implementing an inpatient tobacco cessation service. Poster session presented at the National Conference on Tobacco or Health, Austin, TX, Mar 2017.
47. Rigotti NA, et al. Efficacy of a smoking cessation program for hospital patients. Arch Intern Med. 1997 Dec 8;157:26532660 .

48. Rigotti NA, et al. Sustained care intervention and postdischarge smoking cessation among hospitalized adults: a randomized clinical trial. JAMA. 2014 Aug 20;312:719-728.

49. Shi Y, Warner DO. Surgery as a teachable moment for smoking cessation. Anesthesiology. 2010;112:102-107. 